\title{
Optimized design research of fully enclosed noise barrier
}

\author{
Jiang $\mathrm{Li}^{1}$, YongBin $\mathrm{Li}^{2}$, Jinhua $\mathrm{Tan}^{3, *}$, and Zhimin $\mathrm{Yang}^{3}$ \\ ${ }^{1}$ Shantou local highway affairs center, Guangdong Province, 515000, China \\ ${ }^{2} \mathrm{CCCC}$ Second Highway Consultants Co, Ltd, Wuhan, Hubei Province,430056, China \\ ${ }^{3}$ Road and bridge Dpt. School of Transportation, Wuhan University of Technology, Hubei, 430063, \\ China
}

\begin{abstract}
Fully enclosed noise barrier has been used to prevent and control traffic noise pollution because of its effectiveness. It has become the best solution for controlling environmental noise of high-rise buildings from expressways, urban viaducts and railways that cross the crowded downtown area. However, its high cost has become an important retarding factor on its application and popularization, so reducing the cost become an urgent problem. The research theory, structure frame, sound absorbing and insulating materials are investigated for the fully enclosed noise barrier, so as to provide technical solutions for optimizing the design scheme and reducing the construction cost.
\end{abstract}

\section{Introduction}

The noise barrier has significant advantages on noise reduction, including flexible construction, small occupation and easily maintenance, which is a widely used for traffic noise management all over the world. With the acceleration of urbanization, more and more high-rise buildings have been built, but the semi-closed noise barriers can't work effectively when the residential buildings are higher than 9 floors. The fully enclosed noise barrier has become the best solution on reducing noise for sensitive areas including tall buildings and will be applied more and more in future. At present, fully enclosed noise barriers have been widely used in Canada, Japan and other developed countries. There are also more and more application demand for subways and expressways in Chinese big cities.

Fully enclosed noise barriers should meet the functional requirements of structural safety, noise reduction, durability and aesthetics, so the cost of metal structure frame and sound insulation wall is generally high, which comes to about 100,000 RMB per linear meter, and become the biggest obstacle for its application and popularization. There is an urgent need to research and improve the design of frame and acoustic wall material, so as to reduce the cost.

\footnotetext{
*Corresponding author: 470348706@qq.com
} 


\section{Optimization design theory}

The research on the optimization design theories of noise barriers mainly focuses on the effective application of numerical simulation, the optimization design of sound insulation wall, sound absorbing and insulating materials and the structure of fully enclosed noise barrier.

\subsection{Numerical simulation}

With the development of computer technology, many acoustic simulation software have been developed to model sound performance, such as CADNA /A, Soundplan, Raynoise, etc.

K.M.Li et al. studied the acoustic performance of parallel sound absorbing barrier for one or two parallel facades, and they developed and validated a ray model for calculating complex sound fields in urban areas. Serraris Jasper provided a new and highly visualizing noise prediction method which described the sound path and pressure level with lines and color by Scan\&Paint3D technology. Tikhon Dudiev et al. proposed a comprehensive method for calculating noise barriers to determine the optimum size of the noise barrier, which takes into account both shielded and unshielded noise, including the noise reflected from the road surface and from the opposite noise barrier. D.Duhamel shaped the optimal noise barrier by an optimization method based on the coupling of boundary element method and genetic algorithm. Geng Chuanzhi et al. calculated the insertion loss of the noise barrier by finite-infinite element coupling method based on software ACTRAN. They verified the influence of the width, spacing and protrusion height of structural components on the noise reduction while the noise barrier had circular or triangular protrusions on its surface.

ANSYS can also be used to model the structure and sound insulation function by acoustic-solid coupling simulation of fully enclosed noise barrier, considering sound insulation, absorption and diffraction through the response of various elastic and viscoelastic materials to the incentive sound pressure and the energy absorption performance of their natural vibration.

Because the numerical simulation is not affected by the field conditions and can calculate quickly to get the results under different parameters, they can replace a large number of acoustic noise barrier tests and structural tests, and only need a small number of experiments to determine some key parameters, thus greatly lower the cost.

\subsection{Perforation structure and material optimization}

The materials used for fully enclosed noise barriers are similar to those used for semienclosed noise barriers, including concrete, micro-perforated plate, polyester sound insulation plate, coloured steel sheets, tempered glass, ceramic plate and cement sawdust, etc. Concrete is widely used because of its high strength and lower cost on construction, maintenance, but the sound absorption performance of the traditional concrete is poor, noise reduction effect isn't good enough too. Microporous sound-absorbing materials are widely used because of the large amount of pores on the surface, which lead to great sound energy loss and good sound-absorbing effect.

Micro-perforated plate refers to a large number of holes with diameters less than $1 \mathrm{~mm}$, and there has a appropriate distance from the plate, which not only greatly simplifies the plate structure but also significantly improves its sound absorption coefficient and the range of sound absorption frequency. This theory greatly promotes the development and popularization of fiber free broadband sound absorption structure and micro-perforated diffusing muffler. In 2009, an innovative design and application of perforated closed-foam 
aluminum acoustic board was applied on the noise barriers for the east-west expressway in Shenyang, China. The test results showed that the sound absorption performance could be significantly improved by proper perforating treatment. With the increase of the perforating rate, the peak value of the sound absorption coefficient increases at first and then decreases, shifting to the high frequency gradually.

In 2020, Li Zhanfu put forward a new type of foamed-aluminum noise barrier using the multi-layer composite structure and the main material is made by melting and foaming method. The surface is porous and has a lot of concave-convex and small chambers, which not only absorb noise through surface diffuse reflection, but also dissipate sound energy through microporous structure, thus the sound absorption effect is improved by the cavity structure based on the Helmholtz acoustic resonance.

Qin Xiaochun et al. found that the sonic crystal noise barrier has good noise reduction performance in the band gap range, compared with the vertical composite noise board under the same condition, the noise reduction effect of low frequency is improved by $1 \sim 16 \mathrm{~dB}$ and that of high frequency by $1 \sim 2 \mathrm{~dB}$. But after $1600 \mathrm{~Hz}$, the effect is not as good as the composite board, it is greatly affected by the number of cycles.

As for the sound absorbing material design, the concrete absorbing material was used in the Bird Paradise Ecological Scenic Spot of Shenmao Railway in China for its excellent toughness and higher strength, while the content of PVA fiber is $0.5 \%$, and the content of copper-plated steel fiber is $2 \%$. Engineers used Haoyuan early-strength polycarboxylic acid water reducer in the concrete, so the noise barrier became more compressive and flexible, which reduced the cost too. The noise barrier made of magnesite composite board with straw as filler has the characteristics of noise reduction, environmental protection, durability and good price, etc. It can be designed personally according to local conditions. Foam sound-absorbing material sound insulation board has advantages of easy processing, little pollution, dust resistance, moisture resistance and lower production cost. Fiber absorbent materials have advantages of low density, wide source, low price, good biodegradability and excellent mechanical properties.

\subsection{Structural design optimization}

In the theoretical study of structural design, Tian Xin used Raynoise software to simulate the noise reduction effect of several noise barrier models with different structural forms. Then she improved the top structure of T-shaped noise barrier according to the secondary residual diffusion method and proposed the optimal design scheme. In 2019, Zhu Fei et al. conducted simulated experimental studies on semi-closed noise barriers of various forms, heights and material types based on Soundplan software. The results indicated that the height of noise barriers can effectively affect the effect. The insertion loss increases by 3$4 \mathrm{~dB}$ (A) for each $1 \mathrm{~m}$ increase in the height. The cylindrical barrier top is better than that of the curved barrier for noise reduction effect. W. Shao et al. simulated the performance of random edge barriers based on Rubinowicz diffraction integral numerical simulation.

In practice, the noise barriers of Nanjing Huimin III span bridge is fully enclosed, and it adopts the light steel structure. The sound insulation structure is supported by the steel beam fixed on the steel frame. The steel column is fixed on the anti-collision wall on the two sides of the road by foundation bolts. In the design of the fully enclosed noise barrier of Shanghai rail transit line 6 , the steel frame of the noise barrier is used to suspend the catenary, which can reduce the volume and cost of the project. In order to reduce cost, light steel structure and sound insulation and absorption structure are used to construct long tunnels on Weiqi Road of Yingtian Street in Nanjing, China.

The noise barrier on Yingwuzhou Yangtze River Bridge is made of double-span portal light steel arch frame, while meeting the requirements of traffic noise reduction, natural 
lighting and driving safety, it also integrates with the surrounding architectural landscape to form an urban scenery line for Wuhan, China. In the section of Shenmao Railway Line's Bird Paradise Ecological Scenic Area, a fully enclosed arc-shaped high-strength and hightoughness ultra-thin concrete noise barrier is designed, which has the best aerodynamics performance.

It can reduce the construction cost by painting steel plate and other components with high aluminium-zinc alloy anticorrosive material. The Al- $\mathrm{Zn}$ alloy is made into a scaly powder, and the chrome-free metal coating is made by adding diluent and curing agent. Using this technology, the cost per square meter is reduced by about $10 \%$ compared with the traditional solution of using aluminum alloy to paint the sound absorbing panel.

\section{Conclusion}

To sum up, the optimization design of fully enclosed noise barrier can get breakthroughs in the following aspects:

1) Efficient numerical simulation applications: including modelling the acoustic performance of sound insulation walls, the noise reduction of fully enclosed noise barriers and the whole structure analysis.

2) material design: Choose cost-effective environmental protection materials;

3) structural design: Adopt microhole or multi-layer composite sound absorption barrier with band gap;

4) For the structural design of the fully enclosed noise barrier, the arch shape has the best aerodynamic performance and the variable section composite light steel frame should be recommended;

5) High content aluminium-zinc alloy anticorrosion material should be used in the coating of steel components to lower the cost.

\section{References}

1. K.M.Li and M.K.Law and M.P.Kwok.Absorbent parallel noise barriers in urban environments[J]. Journal of Sound and Vibration, 2008, 315(1-2) : 239-257.

2. Serraris Jasper. Propagation of sound around different noise barrier models using a novel 3D scan based sound visualization technique[J]. Inter Noise and Noise Congress and Conference Proceedings, 2015, 250(4) : 3162-3173.

3. Tikhon Dudiev and Vladimir Tupov. Method of Acoustic Calculation of Traffic Noise Barriers[J]. MATEC Web of Conferences, 2020, 320

4. Geng Chuanzhi,Sun Weina,Zhang Jian.Simulation Analysis of Noise barrier Structure Optimization for Rail Transit [J].Journal of Railway Science and Engineering,2014,11(040;127-134.

5. Tian Xin.Improvement and Optimization Design of Top Structure of Urban Traffic Noise Barrier [D].Beijing Jiaotong University,2014

6. Zhu Fei.Research on Noise Reduction Method of Expressway under Expansion Conditions [D].Jiangsu:Southeast University,2019.

7. Tian Jing.Microperforated plate and small hole injection-professor Ma Dayou's special contribution to acoustics [J].Chinese Journal of Acoustics,2015,40(2):129-133.

8. Fan Lili.Study on Noise Reduction Effect Test and Mechanism of Aluminium foam Noise barrier[D].Northeastern University,2009 
9. Li Zhanfu.Study on Application of New Aluminium Foam Noise barrier in Expressway[J].Highway,2020,65(8):288-290. 